\title{
Review Article \\ Routing in Industrial Wireless Sensor Networks: A Survey
}

\author{
Amal Tiab and Louiza Bouallouche-Medjkoune \\ Laboratory of Modeling and Optimization Systems, Computer Science Department, University of Bejaia, 06000 Bejaia, Algeria \\ Correspondence should be addressed to Amal Tiab; amaltiab@yahoo.fr
}

Received 31 October 2013; Accepted 22 December 2013; Published 12 February 2014

Academic Editors: F. Jiang, Z. Li, and S. Simani

Copyright (C) 2014 A. Tiab and L. Bouallouche-Medjkoune. This is an open access article distributed under the Creative Commons Attribution License, which permits unrestricted use, distribution, and reproduction in any medium, provided the original work is properly cited.

\begin{abstract}
Recently, industrial wireless sensor networks have attracted more attention, due to their large benefits in terms of faster installation, cost savings, and flexibility. Nevertheless, the acceptance of wireless sensor networks by the industrial community is not without its difficulties. In fact, several research efforts have been made in this field and a number of state-of-the-art reviews exist, presenting the current standards, the challenges, and the design principles. However, a comprehensive review on routing protocols appears to be missing. In this paper, we give a survey on routing protocols for industrial monitoring applications of the wireless sensor networks technology, and we present their limitations and weaknesses according to the industrial requirements.
\end{abstract}

\section{Introduction}

Industrial wireless sensor networks (IWSNs) have been gaining acceptance during the last decade, largely due to the greatly increased flexibility, lower cost, and scalability that they have been shown to provide [1]. Nevertheless, quite a few issues have not been addressed properly in existing research work in this field. For example, a very challenging problem like the design of routing protocols in the industrial wireless sensor networks still needs to be explored.

Routing is a key process to be considered in the industrial wireless sensor networks due to their inherent characteristics. In such environment, due to the limited wireless transmissions range of each node, it may be necessary for one sensor node to ask for the aid of other sensor nodes in forwarding a packet to its destination, usually the sink [2]. Consequently, the routing protocol in industrial wireless sensor networks should not only facilitate the data transmission, but also consider sensor constraints and to provide reliability and latency requirements since routing decisions can impact the network lifetime, the packet delivery rates, and the end-toend packet delays.

A number of exiting surveys in the industrial wireless sensor networks field present the industrial challenges and technical approaches or comparisons of the current standards $[3,4]$. However, a comprehensive review on routing protocols appears to be missing. The aim of this paper is to analyze the exiting routing protocols proposed in the literature for a specific class of industrial applications which is monitoring and to present their limitations and weaknesses according to the industrial routing requirements.

The rest of the paper is structured as follows. In Section 2, we first present the main challenges in the industrial wireless sensor networks and give an overview on the industrial applications classes, we also present a typical network topology of the industrial applications, and finally we summarize some of the requirements in the industrial environment to design a routing protocol in wireless sensor networks. In Section 3, we present a state of the art of the routing protocols for the industrial monitoring applications of the Wireless Sensor Networks and we conclude our work in Section 4.

\section{Industrial Wireless Sensor Networks}

Industrial applications represent a class of sensor network applications with enormous potential benefits for engineering communities. Many of the attempts aim at eliminating cabling in the industry environment, among which wireless sensor networks are a novel and promising technology [5]. In this section, we present a classification of the industrial applications [6]. We also present the appropriate network topology suggested for industrial monitoring applications. Finally, we highlight the basic requirements for designing an efficient 
routing protocol for industrial monitoring applications of the wireless sensor networks; see Table 2 [7].

2.1. The Main Challenges in the Industrial Wireless Sensor Networks. The biggest challenges for the realization of the IWSNs can be outlined as follows [8].

(i) The harsh industrial environmental conditions may cause a portion of industrial sensor nodes to malfunction [9], since, in such environments, sensors may be subject to RF (radio frequency) interference, highly caustic or corrosive environments, high humidity levels, vibrations, dirt and dust, or other conditions that challenge performance [10].

(ii) Limited resources: the design and implementation of IWSNs are constrained by the limited physical size of the sensor nodes; they have limited battery energy supply. At the same time, they have limited memories and restricted computational capabilities [11].

(iii) Quality-of-service requirements, due to the diverse range of applications that can be found in industrial scenarios and their differing requirements, there is a need for quality of service provisioning so that the technology can be successfully adopted, especially in terms of reliability, energy management, and message transmission delays. The QoS provided by IWSNs refers to the accuracy between the data reported to the sink node and what is actually occurring in the industrial environment.

(iv) Data redundancy, sensor observations are highly correlated in the space domain, because of the high density in the network topology. Moreover, the nature of the physical phenomenon constitutes the temporal correlation between each consecutive observation of the sensor node.

(v) Packet errors and variable-link capacity, in IWSNs, wireless links exhibit widely varying characteristics over time and space due to noisy environment. Thus, capacity and delay attainable at each link are location dependent and vary continuously, making QoS provisioning a challenging task.

(vi) Large-scale deployment and ad hoc architecture, most IWSNs contain a large number of sensor nodes (hundreds to thousands or even more), which might be spread randomly over the deployment field. Moreover, the lack of predetermined network infrastructure necessitates the IWSNs to establish connections and maintain network connectivity autonomously.

2.2. Industrial Applications. Due the diversity of the industrial applications, they are classified into three categories and six classes by industrial market, as presented in Table 1 .

Class 0 "emergency action" includes safety-related actions that are critical to both personnel and plant. Most safety functions are performed through dedicated wired networks to limit both failure and vulnerability to external events or attack. Examples are safety interlock, emergency shutdown, and fire control.

Class 1 "closed loop regulatory control" includes motor and axis control as well as primary flow and pressure control.

Class 2 "closed loop supervisory control" usually has long time constants, with latency of communications measured in seconds to minutes. Example is equipment selection.

Class 3 "open loop control" includes actions where an operator, rather than a machine, closes the loop between input and output. Latency for this class of action is human scale, measured in seconds to minutes.

Class 4 "monitoring with short-term operational consequences" includes high-limit and low-limit alarms and other information that might evoke more verification. Latency for this class of information is typically low, measured in minutes or even hours.

Class 5 "monitoring without immediate operational consequences" includes objects without strong latency requirements. Some, like sequence of events, require high reliability; others, like reports of slowly-changing information of low economic value, do not need to be so reliable since loss of a few consecutive samples may be unimportant.

A recent study [12], presented by the International Society of Automation [6], identified that industrial users are interested in deploying wireless networks for the less critical applications such as the monitoring classes 4 and 5, where determinism is not required and higher latencies can be tolerated. In fact, when considering deploying a wireless sensor network in a factory installation, $88.8 \%$ of interested parties "users and vendors" identified a strong preference for monitoring applications. Control applications were only considered by $13 \%$ of those companies surveyed. So, our main focus in this work is on the monitoring applications, where the successful delivery of data is of a paramount interest and the acceptable delays for the applications are in the order of seconds to minutes.

\subsection{Network Topology of Industrial Monitoring Applications.} Although the network topology is difficult to generalize in some kind of applications, the appropriate network topology suggested for the industrial monitoring applications is the mesh topology or a hybrid of the star and the mesh topology [7]. According to the networking working group (NWG) of the internet engineering task force (IETF) [5], typical industrial scenarios may have multiple sinks with the number of sinks being far smaller than the total number of nodes. The network may be composed of 10 to 200 nodes and usually the maximum number of hops to reach the sink from any source is 20 hops. It is assumed that the nodes themselves will provide routing capability for the network. In addition, they should be small and easily deployed with reduced battery and memory capacity. They should be able to operate in a wide range of environmental conditions found in industrial scenarios. Also, it is generally expected that nodes with routing capabilities will be stationary as well as the sinks that will be connected to the backbone. An example of a typical topology of industrial monitoring applications presented by ISA SP100.11 in [13] is illustrated in Figure 1. 
TABLE 1: Industrial applications classification.

\begin{tabular}{lcll}
\hline Category & Class & Application & Description \\
\hline Safety & 0 & Emergency action & Always critical \\
Control & 1 & Closed loop regulatory control & Often critical \\
Control & 2 & Closed loop supervisory control & Usually noncritical \\
Control & 3 & Open loop control & Human in the loop \\
Monitoring & 4 & Alerting & $\begin{array}{l}\text { Short-term operational consequence. For } \\
\text { example, event-based maintenance } \\
\text { No immediate operational consequence. For } \\
\text { Monitoring }\end{array}$ \\
& 5 & Logging and downloading/uploading & history collection, sequence of events, \\
& & &
\end{tabular}

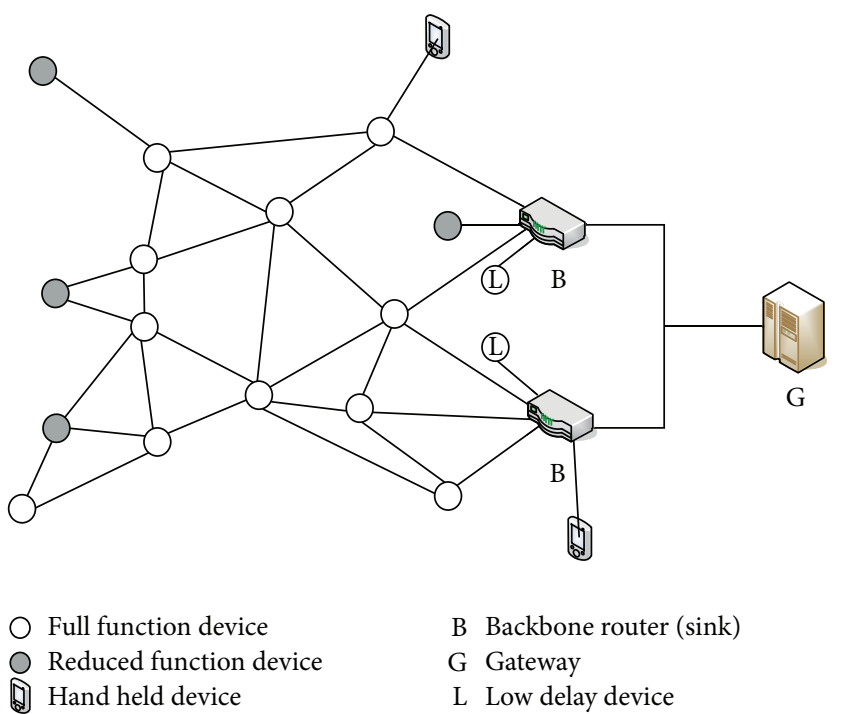

FIGURE 1: ISA SP100.11a basic network.

2.4. Routing Requirements for the Industrial Wireless Sensor Networks. In this part, we highlight some of the issues that must be taken into consideration when designing routing protocols for industrial monitoring applications of wireless sensor networks, from the viewpoint of the ISA100.11 standardization committee [6] and the ROLL working group [14]. The basic requirements for an efficient routing protocol considered for wireless sensor networks in industrial monitoring applications expect that the routing protocol is able to

(i) provide reliability and soft latency requirements "seconds to minutes,"

(ii) compute multiple paths of not-necessarily-equal cost toward a given destination so as to enable loadbalancing across a variety of paths,

(iii) support different metric types for each link used to compute the path according to some objective function for example, minimize latency and depending on the nature of the traffic,

(iv) support the ability to recompute paths based on the network-layer abs tractions of the underlying link attributes/metrics that may change dynamically, (v) consider node-constrained routing. This includes power and memory, as well as constraints placed on the device by the user, such as battery life,

(vi) distribute sufficient information about the link failures to enable traffic to be routed such that all service requirements especially latency continue to be met,

(vii) be easy to deploy and manage,

(viii) limit the risk incurred by one node being compromised, for instance by proposing a noncongruent path for a given route and balancing the traffic across the network. It must be noted that security considerations are outside of the scope of our work.

The next section presents the state of the art of the exiting routing protocols for the industrial monitoring applications of Wireless Sensor Networks.

\section{Routing Protocols in Industrial Wireless Sensor Networks}

We present here the exiting routing protocols in the industrial wireless sensor networks, especially routing protocols for the monitoring applications, where the successful delivery of data is of a paramount interest and the acceptable delays for the applications are in the order of seconds to minutes. We present their limitations and weaknesses according to the industrial routing requirements presented in Section 2.

Heo et al. proposed in [15] EARQ (energy aware routing for real-time and reliable communication in wireless industrial sensor networks). It is a location-based proactive routing protocol that aims to maintain an ongoing routing table. In EARQ, a node estimates the energy cost, the delay and the reliability of a path toward the sink node, based only on the information from neighboring nodes. It selects a path that expends less energy among paths that deliver a packet in time. Sometimes, it selects a path that expends more energy than the optimal path, because the path is randomly selected, according to a probability. The deadline, which is the maximum tolerable packet delay, is estimated based on the density of the sensor nodes and the radio range. In addition, EARQ sends a redundant packet via an alternate path if the reliability of a path is less than a predefined value. However, the number of packets in the network increases and it can be congestion or increased energy expenditure. 
TABLE 2: Routing protocols for industrial wireless sensor networks.

Protocol
EARQ: energy aware routing for real time
and reliable communication in wireless
industrial sensor networks [15]

Strengths

Provides reliability, reduced delay, and energy efficiency
Weaknesses

(i) Requires global accurate positioning information to perform the routing

(ii) Results in control message overhead and does not consider the buffer size limitation of the sensor nodes

(i) Results in data redundancy

(ii) Does not consider delay

(iii) Does not motion how to measure routing metrics and does not consider the buffer size limitation of the sensor nodes

Uses a single path for routing the data packets and does not consider the inherent characteristics of sensor nodes, which are energy and buffer size limitations

A reliable and energy efficient routing protocol [16]

Provides reliability and reduces the number of control packets (i) Provides reliability, low latency, and low complexity

(ii) No duplicates and no outdated data packets

(iii) Guaranteeing packet progress towards the sink

(i) Provides reliability, low latency and service differentiation

(ii) Considers energy and buffer limitations adaptive to any multipath routing protocol

networks [13]

POCTP: Pareto optimal collection tree protocol for industrial monitoring WSNs [20]

Enhancing real time delivery of gradient routing for industrial wireless sensor networks [21] (i) Requires accurate positioning information to perform the routing tasks

(ii) Not energy efficient and does not consider the buffer size limitations

(i) This algorithm does not adopt an effective method for delay calculating; it only considers the minimum number of hops towards the destination

(ii) Needs some time to converge

(i) Results on control messages overhead

(ii) Does not consider energy and buffer size limitations

(i) Use a single path for routing data packets (ii) Does not consider reliability that must be taken into consideration in Industrial Wireless Sensor Networks and does not consider the buffer size limitation
This protocol requires global accurate positioning information to perform the routing tasks and to calculate some of the route selection metrics, which cannot be reliably achieved in indoor scenarios. The location information can be obtained by GPS or localization protocols for estimating the location of a node. This protocol does not consider the inherent properties of WSNs such as the buffer size limitation of the sensor nodes.

Wang et al. presented in [14] a reliable routing protocol based on deterministic schedule for wireless industrial networks. The aim of this protocol is to provide reliability and to meet the special requirement of deterministic scheduling for industrial applications. Moreover, the protocol has the advantages of energy saving and the ability of packets aggregation. The authors developed an improved routing mechanism with feedback and redundancy and proposed a deterministic routing algorithm for both cluster and mesh networks. The algorithm supports deterministic schedule, redundancy, and VCR (virtual communication relations hip) for aggregation. It applies and improves $\mathrm{k}$-shortest path algorithm [15]. However, more comprehensive details on how to measure routing metrics are not motioned.
Kim and Ngo proposed in [16] a reliable and energy efficient routing protocol in industrial wireless sensor networks considering a path with highest end-to-end PRR (packet reception rate) value for transmitting data packets. Data packets are periodically transmitted by the source node over the selected path. Actually, the route establishment mechanism in the proposed protocol is the same as AODV [17]. It is based on the exchanging of RREQ (route request)RREP (route replay)_DATA packets between the source and the destination. The differences between the proposed protocol and AODV are as follows: each node maintains a PRR value, which is the product of PRR values of the links which composed the reverse path from this node to the source, each RREQ packet contains PRR value of the node that sends out the RREQ and prevId (the identifier of the previous node). Each node calculates the delay time when receiving a RREQ from its neighboring nodes. During the delay time, if a node receives a RREQ sent from a node that has the same prevId as itself, then this node stops rebroadcasting RREQ again. The destination waits for an interval of time to collect multiple the RREQ packets. When this time is expired, the destination selects the RREQ packet 
that has the highest PRR value to transmit RREP. The source, when receiving this RREP, will transmit the data through the established path. The proposed protocol uses only a single path for routing the data packets. It also does not consider the inherent characteristics of the sensor nodes, which are the energy and the buffer size limitations.

Barac et al. presented in [18] a lightweight routing protocol for industrial wireless sensor and actuator networks. They recycled the flooding concept by introducing minor modifications to its generic form in order to exploit its inherently good properties and make it utilizable for uplink in Industrial Wireless Sensor Network applications. The approach is distributed, where each intermediate node independently decides whether to retransmit or discard the received packet. All the information necessary for making the forwarding decision is extracted or derived from the content of the data packets, so there are no control messages exchanged between the nodes. Every packet contains a unique application payload identifier and every node should store the identifiers of seen packets in order to handle the duplicates. A Packet age, TTL (time-to-live) field is checked at every hop and outdated packets are discarded. The approach is location based: the authors used the concept of node weight introduced in [19], in order to make a packet progress towards the sink at every hop. This protocol is capable of delivering data efficiently with low latency and significantly less complexity, but the latency is highly correlated with the topology size and the sensor refresh rate. It is also not energy efficient and does not consider the buffer size limitation of the sensor nodes.

Villaverde et al. proposed in [13] the InRout route Selection algorithm, an adaptive multimetric based route selection algorithm that uses Q-learning to choose the best routes based on the current network conditions and application settings, which can be used with any underlying multipath routing protocol. InRout considers the inherent restrictions and challenges imposed by WSNs with a route differentiation in order to satisfy the needs of the industrial applications like required PER, delay, or energy. InRout is a route selection algorithm that depends on the multipath routing protocols efficiency. For instance, in the case of a route or node failure, it is the role of the underlying routing protocol to discover alternate routes and perform route maintenance. This algorithm does not adopt an effective method for delay calculation; it only considers the minimum number of hops towards the destination and needs some time to converge.

$\mathrm{Wu}$ et al. proposed in [20] POCTP (Pareto optimal collection tree protocol for industrial monitoring WSNs) in order to meet multiobjective transmission requirements in industrial monitoring WSNs. This work improved the original CTP [8], that is, a tree based collection protocol where sensor nodes form a set of routing trees with sink node as the roots, and the nodes generate routes using routing gradient measurement. However, CTP (collection tree protocol) is the best effort mechanism, so it does not provide low transmission delay. POCTP uses a multiobjective optimization Pareto based approach to ensure QoS (e.g., transmission throughput, delay, loss of packets). It uses dynamic distributed optimization to select the best routes in order to send sensed data to the sink node within a due time and with the highest reliability. Unique Pareto route set based routing framework, including link quality estimator, routing setting up, and routing engine, is put forwarded grounding on the CTP [8]. This protocol provides real time and reliable data transmission for the industrial monitoring WSNs; it is illustrated by a comprehensive and reasonable hierarchical Petri net based verification model. However, it leads to increase the control messages overhead and does not consider the energy and the buffer size limitations.

Quang proposed in [21] a two-hop neighbor informationbased gradient routing to enhance real-time performance with energy efficiency. The proposed scheme combines THVR (two-hop velocity-based routing) [22] and a gradientbased network. So the optimal path is archived in terms of the number of hops to the sink instead of the distance which reduces energy consumption. Additionally, it adopts a selective ACK (acknowledgment) scheme to update twohop information. To adapt gradient-based networks, this algorithm uses the schemes proposed in IETF ROLL [14, 23]. The schemes are composed of gradient setup, height calculation, and forwarding techniques. In the setup phase, the sink broadcasts a packet containing a counter set to 1 . Upon receiving the packet, a node sets its height equal to the counter in the packet (increases the counter by 1 ) and forwards the packet. The sink sets its height to 0 . The heights of other nodes are equal to the smallest number of hops to the sink. Each node calculates joint parameters. A node compares the joint parameters of its neighboring nodes and selects a neighbor to relay its packets to the sink. The proposed algorithm selects the optimal single path based on the delay and does not consider the reliability that must be taken into consideration in the industrial wireless sensor networks.

\section{Conclusion and Future Work}

In this paper, we have surveyed the state of the art of recent research results on routing protocols for industrial wireless sensor networks. Special attention has been devoted to the routing protocols for monitoring applications. We presented an overview of the industrial application classes and typical network topology of the industrial monitoring applications. We also highlighted some of the requirements for designing routing protocols in the industrial wireless sensor networks. Finally, we summarized the strengths and the weaknesses of the above investigated routing protocols.

As mentioned in Section 2.3, the main challenges in the design of routing protocols for the industrial wireless sensor networks are the reliability, the soft latency and the energy efficiency. Moreover, a routing protocol in such applications is expected to be able to compute multiple paths in order to provide load balancing, fault tolerance, bandwidth aggregation, and reduced delay. Considering node-constrained routing is also a main routing requirement; this includes power and memory. Although the exiting routing protocols presented advantages in providing reliability, reducing delays, and data aggregation, they have deficits that make them do not comply with the industrial routing requirements. For instance, in 
terms of using single path routing or requiring global accurate positioning information, this can not be reliably achieved in indoor scenarios. Another interesting point is that most of the solutions proposed in the literature assume data redundancy and the exchange of routing tables and messages used for network self-recovery or node-discovery poses a significant overhead. Some existing routing methodologies do neither provide both reliability nor reduced delay. Moreover, there are other protocols that do not consider sensor nodes limitations or application requirements, which makes them unsuitable for realistic scenarios. Finally, as can be observed, these schemes treat wireless links as point-to-point wired links and ignore the unique broadcast nature of the wireless medium, which can be utilized to improve link reliability and system throughput by routing packets through multiple routes dynamically; this is known as opportunistic routing $(\mathrm{OR})$. We are persuaded that opportunistic routing $(\mathrm{OR})$, will get an even greater importance and attention within the routing for industrial wireless sensor networks since this new routing paradigm may present several merits to the IWSNs, by increasing reliability, that is, a main IWSN's challenge; OR transmits a packet through any possible link rather than one specified link. In other words, it acts like has additional backup links and the probability of transmission failure is reduced [24]. It also provides the highest throughput, since all neighboring nodes that are closer to the destination may overhear a data packet and may be a candidate to forward the packet to its destination. Increase transmission range: OR considers all possible links, including good quality shortranged links and poor quality long-ranged links, within one transmission; therefore, a transmission may directly jump to the farthest relay which successfully receives the packet. Consequently, performance can be improved [24].

\section{Conflict of Interests}

The authors declare that there is no conflict of interests regarding the publication of this paper.

\section{References}

[1] J. Åkerberg, M. Gidlund, and M. Bjorkman, "Future research challenges in wireless sensor and actuator networks targeting industrial automation," in Proceedings of the 9th IEEE International Conference on Industrial Informatics (INDIN '11), pp. 410415, July 2011.

[2] I. F. Akyildiz, W. Su, Y. Sankarasubramaniam, and E. Cayirci, "Wireless sensor networks: a survey," Computer Networks, vol. 38, no. 4, pp. 393-422, 2002.

[3] V. C. Gungor and G. P. Hancke, "Industrial wireless sensor networks: challenges, design principles, and technical approaches," IEEE Transactions on Industrial Electronics, vol. 56, no. 10, pp. 4258-4265, 2009.

[4] R. D. Team RPL, "Routing protocol for low power and lossy networks IETF ROLLWG. IETF Internet-Draft," February 2009.

[5] M. Soyturk and A. Altir, "Source-initiated geograp hical data flow for wireless ad hoc and sensor networks," in Proceedings of the IEEE Annual Wireless and Microwave Technology Conference (WAM ICON '06), pp. 1-5, December 2006.
[6] ISA-100.11a-2009, Wireless SyStems for Industrial Automation: Process Control and Related Applications, 2009.

[7] Industrial Routing Requirements in Low Power and Lossy Networks, Work In Progress Internet Draft, Networking Working Group, Internet Engineering Task Force, 2009.

[8] Fonseca, R. Gnawali, O. Jamieson, K. Kim, S. Levis P, and A. Woo, "The collection tree p rotocol (CTP)," Tiny OS TEP, vol. 123, p. 2, 2006.

[9] V. C. Gungor, M. C. Vuran, and O. B. Akan, "On the cross-layer interactions between congestion and contention in wireless sensor and actor networks," Ad Hoc Networks, vol. 5, no. 6, pp. 897-909, 2007.

[10] U.S. Department of Energy, "Industrial wireless technology for the 21st century," Office of Energy and Renewable Energy Report, 2002.

[11] V. C. Gungor and F. C. Lambert, "A survey on communication networks for electric system automation," Computer Networks, vol. 50, no. 7, pp. 877-897, 2006.

[12] X. Shen, Z. Wang, and Y. Sun, "Wireless sensor networks for industrial applications," in Proceedings of the 5th World Congress on Intelligent Control and Automation (WCICA '04), pp. 36363640, June 2004.

[13] B. C. Villaverde, S. Rea, and D. Pesch, "InRout-a QoS aware route selection algorithm for industrial wireless sensor networks," Ad Hoc Networks, vol. 10, no. 3, pp. 458-478, 2012.

[14] H. Wang, G. Ge, J. Chen, and P. Wang, "A reliable routing protocol based on deterministic schedule for wireless industrial networks," in Proceedings of the 3rd IEEE International Conference on Computer Science and Information Technology (ICCSIT '10), pp. 368-372, July 2010.

[15] J. Heo, J. Hong, and Y. Cho, "EARQ: energy aware routing for real-time and reliable communication in wireless industrial sensor networks," IEEE Transactions on Industrial Informatics, vol. 5, no. 1, pp. 3-11, 2009.

[16] M. K. Kim and H. P. Ngo, "A reliable and energy efficient routing protocol in industrial wireless sensor networks," in Proceedings of the 4th Annual International Conference on Advanced Technologies for Communications (ATC '11), pp. 3235, August 2011.

[17] C. E. Perkins, E. M. Belding-Royer, and R. DasS, "Ad hoc Ondemand Distance Vector (AODV) routing," Internet Experimental Erimental RFC 3561, 2003.

[18] F. Barac, J. Åkerberg, and M. Gidlund, "A lightweight routing protocol for Industrial Wireless Sensor and Actuator Networks," in Proceedings of the 37th Annual Conference of the IEEE Industrial Electronics Society (IECON '11), pp. 2980-2985, November 2011.

[19] Z. S. P. Thubert, T. Watteyne, and D. Barthel, "Lin Routing Fundamentals," IETF ROLL, IETF Internet-Draft, April 2009.

[20] Y.-Z. Wu, D.-P. Quan, and H.-G. Han, "Pareto Optimal Collection Tree Protocol for industrial monitoring WSNs," in Proceedings of the IEEE Global Communications Conference (GLOBECOM '11), pp. 508-512, Houston, Tex, USA, December 2011.

[21] P. T. A. Quang and D.-S. Kim, "Enhancing real-time delivery of gradient routing for industrial wireless sensor networks," IEEE Transactions on Industrial Informatics, vol. 8, no. 1, pp. 61-68, 2012.

[22] Y. Li, C. S. Chen, Y.-Q. Song, Z. Wang, and Y. Sun, "Enhancing Real-time delivery in wireless sensor networks with two-hop information," IEEE Transactions on Industrial Informatics, vol. 5, no. 2, pp. 113-122, 2009. 
[23] P. Sereiko and D. Caro, "What process control professionals think about wireless: analysis of an ISA 100 and control magazine survey," in Proceedings of the ISA Expo Conference, pp. 2328-2359, Houston, Tex, USA, October 2007.

[24] C.-J. Hsu, H.-I. Liu, and W. K. G. Seah, "Opportunistic routing-a review and the challenges ahead," Computer Networks, vol. 55, no. 15, pp. 3592-3603, 2011. 

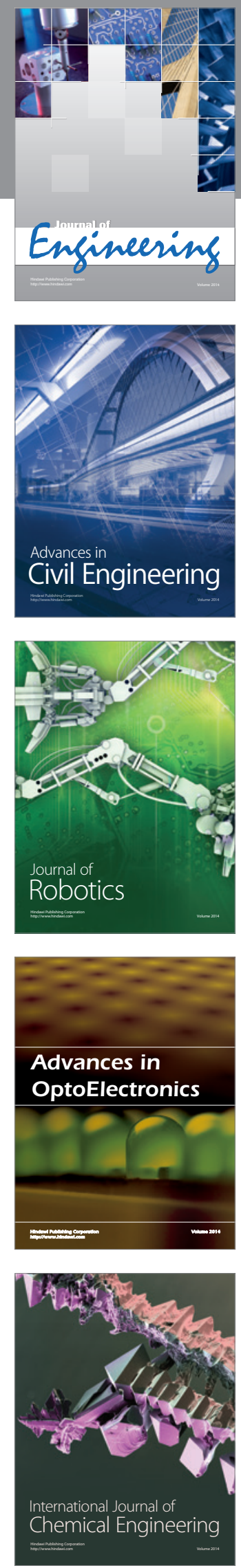

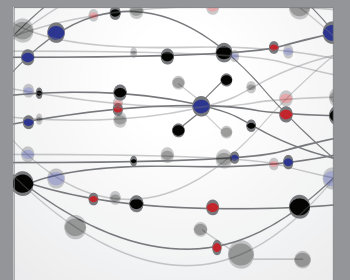

The Scientific World Journal
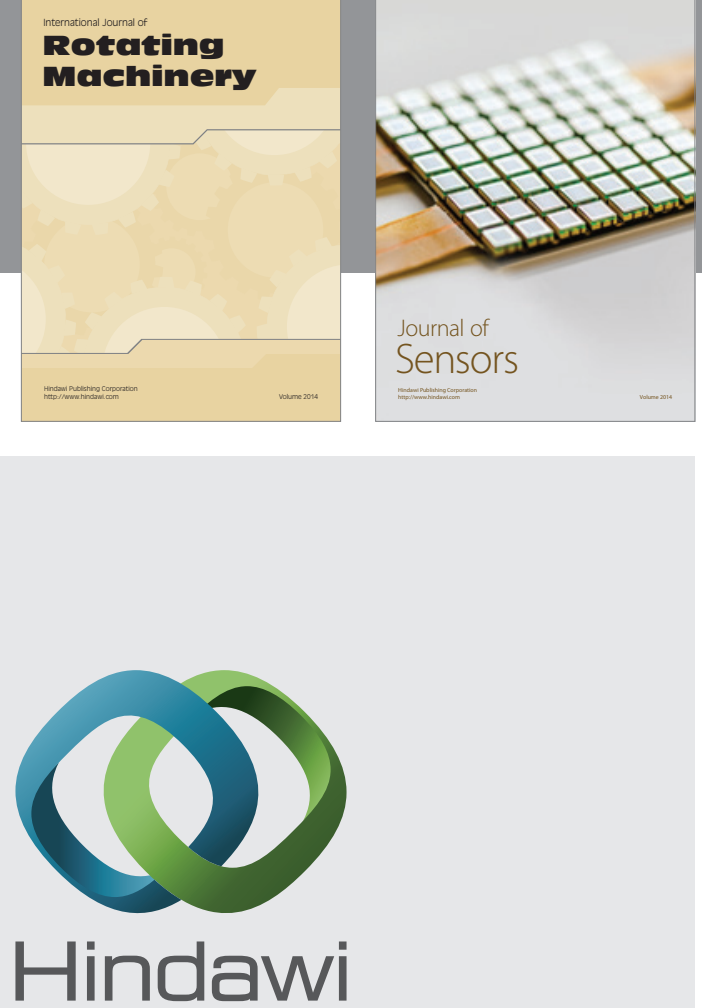

Submit your manuscripts at http://www.hindawi.com
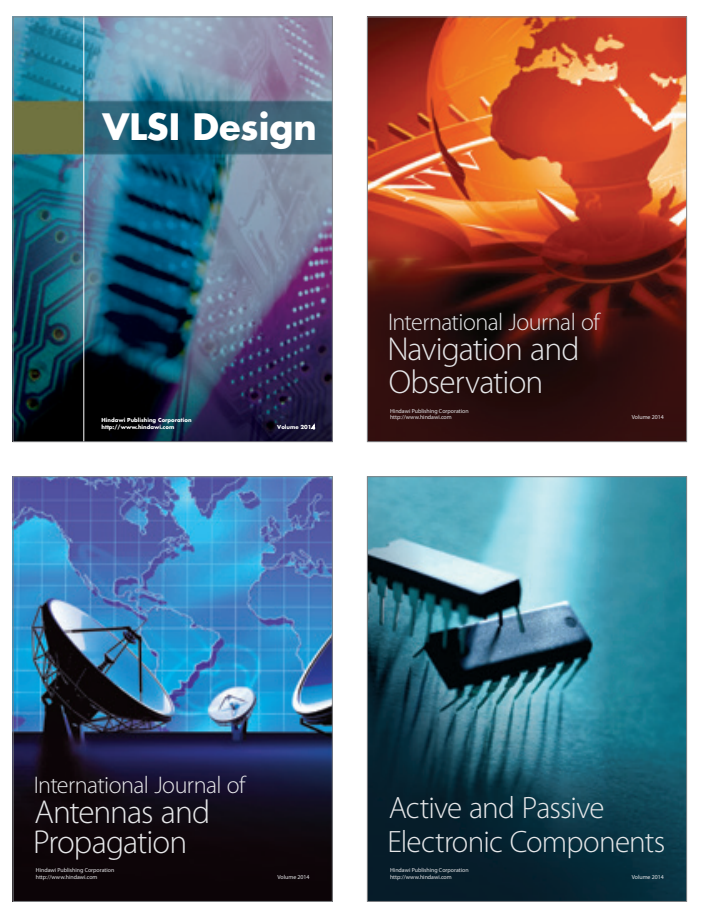
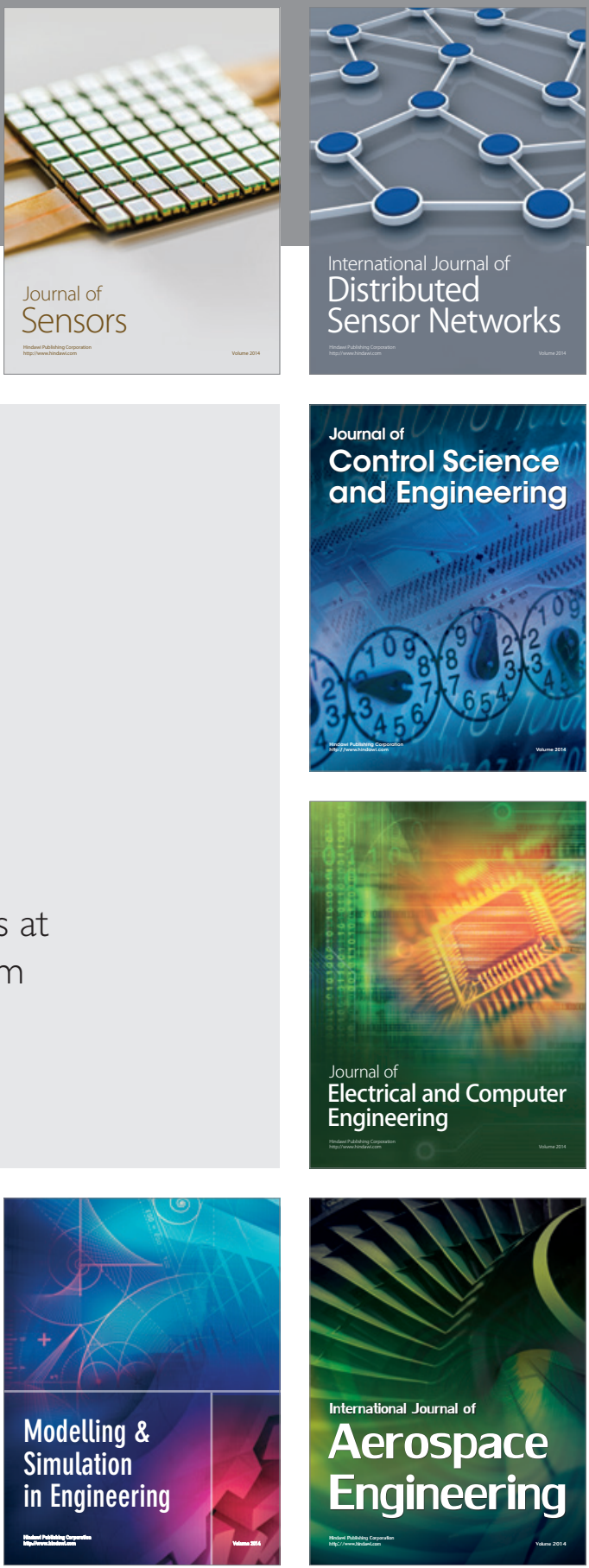

Journal of

Control Science

and Engineering
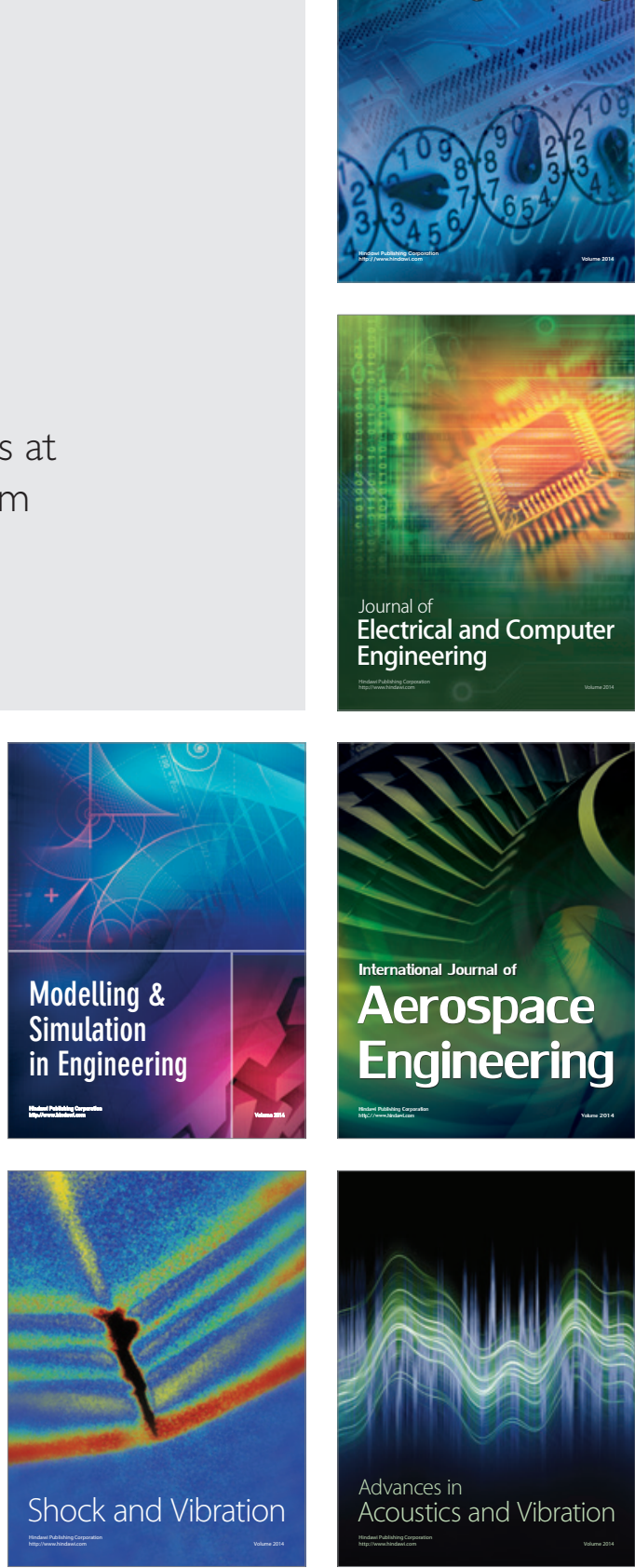